\title{
Clinical characteristics and treatment of delirium in palliative care settings
}

\author{
Filipe Portela Millinger · Matthäus Fellinger $(D$
}

Received: 17 May 2020 / Accepted: 21 July 2020 / Published online: 14 August 2020

(C) The Author(s) 2020

\begin{abstract}
Summary Delirium is commonly seen in palliative care. It usually develops over a short period of time and is characterized by a disturbance of attention and awareness. As delirium is associated with increased mortality, prevention and early identification of this severe neurocognitive disorder is of high clinical relevance. This paper provides a brief overview of risk factors, preventive measures, current screening and diagnostic procedures, as well as nonpharmacological and pharmacological treatment options of delirium in the palliative care setting.
\end{abstract}

Keywords Confusion - Diagnosis · Symptom assessment · Prevention and control · Drug therapy

\section{Delirium is frequent(ly missed) in the palliative care setting}

Delirium is a neurocognitive disorder commonly seen in palliative care and the critically ill. According to an updated systematic review up to three quarters of palliative inpatients are affected, with a pooled point prevalence on admission of $35 \%$ and a prevalence of up to $88 \%$ prior to death [1]. The illness is generally associated with an increased short-term as well as long-term mortality and prolonged stay in intensive care [2, 3]. Furthermore, delirium is a predictor of adverse outcome [4]. Apart from the exorbitant financial burden there is also evidence that delirium causes enormous distress for caregivers [5, 6]. Despite its severity and frequency, delirium is still underdiagnosed and often missed [7]. Timely diagnosis

\footnotetext{
F. Portela Millinger, MD $\cdot$ M. Fellinger, MD $(\bowtie)$

Clinical Division of Social Psychiatry, Department for

Psychiatry and Psychotherapy, Medical University of Vienna,

Waehringer Guertel 18-20, 1090 Vienna, Austria

matthaeus.fellinger@meduniwien.ac.at
}

of this acute condition is essential as it is potentially reversible, e.g. in $20-50 \%$ of advanced cancer patients [8]. However, the diagnosis depends to a great extent on the expertise of the staff, the diagnostic tools and the subtype of delirium [7,9]. Thus, the aim of this paper is to provide a brief overview of risk factors and preventive measures, current diagnostic procedures, as well as nonpharmacological and pharmacological treatment options for delirium in palliative care that have to be considered depending on patient's state and treatment goals.

\section{Evaluate changes in attention and awareness}

According to the eleventh revision of the International Classification of Diseases (ICD-11), delirium is primarily characterized by disturbed attention and awareness. It usually develops over a short period of time and shows fluctuations over the course of a day with more severe symptoms during the evening and the night. Furthermore, it is often accompanied by cognitive impairment such as disorientation, memory deficit, or impairment in language, visuospatial ability, or perception. In addition, disturbance of the sleep-wake cycle may be present [10]. An overview of the diagnostic characteristics of delirium according to ICD-11 and the Diagnostic and Statistical Manual of Mental Disorders, Fifth Edition (DSM-5) are shown in Table 1. Unlike the ICD-11, the DSM-5 also makes the distinction between hyperactive, hypoactive and mixed delirium [11]. While hyperactive and mixed subtypes are more precisely and easily identified due to excessive psychomotor activity, hypoactive delirium is often overlooked, although it may be predominant in palliative care with approximately $40 \%$ of delirium patients [1].

It is crucial to identify delirium as soon as possible, as delayed treatment may result in worse outcomes 
Table 1 Diagnostic characteristics of delirium according to ICD-11 and DSM-5 [10, 11]

ICD-11

Delirium is characterized by disturbed attention (i.e. reduced ability to direct, focus, sustain, and shift attention) and awareness (i.e. reduced orientation to the environment) that develops over a short period of time and tends to fluctuate during the course of a day, accompanied by other cognitive impairment such as memory deficit, disorientation, or impairment in language, visuospatial ability, or perception. Disturbance of the sleep-wake cycle (reduced arousal of acute onset or total sleep loss with reversal of the sleep-wake cycle) may also be present. The symptoms are attributable to a disorder or disease not classified under mental and behavioural disorders or to substance intoxication or withdrawal or to a medication
DSM-5

A. Disturbance in attention (i.e. reduced ability to direct, focus, sustain, and shift attention) and awareness (reduced orientation to the environment).

B. The disturbance develops over a short period of time (usually hours to a few days), represents a change from baseline attention and awareness, and tends to fluctuate in severity during the course of a day.

C. An additional disturbance in cognition (e.g. memory deficit, disorientation, language, visuospatial ability, or perception).

D. The disturbances in Criteria $\mathrm{A}$ and $\mathrm{C}$ are not better explained by another pre-existing, established, or evolving neurocognitive disorder and do not occur in the context of a severely reduced level of arousal, such as coma.

E. There is evidence from the history, physical examination, or laboratory findings that the disturbance is a direct physiological consequence of another medical condition, substance intoxication or withdrawa (i.e. due to a drug of abuse or to a medication), or exposure to a toxin, or is due to multiple aetiologies. Specify if:

Hyperactive: The individual has a hyperactive level of psychomotor activity that may be accompanied by mood lability, agitation, and/or refusal to cooperate with medical care.

Hypoactive: The individual has a hypoactive level of psychomotor activity that may be accompanied by sluggishness and lethargy that approaches stupor.

Mixed level of activity: The individual has a normal level of psychomotor activity even though attention and awareness are disturbed. Also includes individuals whose activity level rapidly fluctuates

Table 2 Guide to preventive, diagnostic and treatment measures (without claiming to be complete)

\begin{tabular}{|c|c|c|}
\hline Steps & What could be done & Examples \\
\hline $\begin{array}{l}\text { Identify patients } \\
\text { at-risk of delirium }\end{array}$ & $\begin{array}{l}\text { Information for the team, patients, care givers regarding } \\
\text { delirium, its risk factors and its preventive measures }\end{array}$ & Risk factors: age, CNS tumour, comorbidity, polypharmacy \\
\hline $\begin{array}{l}\text { Screen for delir- } \\
\text { ium }\end{array}$ & Implement screening tools & CAM \\
\hline Diagnose & Diagnostic characteristics of DSM- 5 and ICD-11 & Be aware of hypoactive delirium \\
\hline \multirow{3}{*}{$\begin{array}{l}\text { Assess } \\
\text { (depending on } \\
\text { patient's state and } \\
\text { treatment goals) }\end{array}$} & Clinical/physical assessment & Blood pressure, pulse, auscultation, temperature, skin turgor, ... \\
\hline & Review medication and rule out further causes & DEL-FINE score/I WATCH DEATH \\
\hline & $\begin{array}{l}\text { Consider appropriate laboratory and radiological investiga- } \\
\text { tions }\end{array}$ & Chest X-ray, urine analysis/urine culture, electrolytes \\
\hline \multirow{3}{*}{$\begin{array}{l}\text { Treat } \\
\text { (depending on } \\
\text { patient's state and } \\
\text { treatment goals) }\end{array}$} & Treatment of potentially reversible causes & According to respective guideline \\
\hline & Non-pharmacological interventions & $\begin{array}{l}\text { Support orientation } \\
\text { Sleep logs and rhythmic activities } \\
\text { Physical therapy } \\
\text { Hearing and visual aids } \\
\text { Sufficient fluid, balance } \\
\text { Integration of relatives }\end{array}$ \\
\hline & Pharmacological treatment & Short-term administration of antipsychotics at the lowest effective \\
\hline
\end{tabular}

DSM-5Diagnostic and Statistical Manual of Mental Disorders, Fifth Edition, ICD-11 eleventh revision of the International Classification of Diseases, CAM Confusion Assessment Method, I WATCH DEATH Infections, Withdrawal, Acute metabolic causes, Trauma, CNS pathology, Hypoxia, Deficiencies, Endocrinopathies, Acute vascular, Toxins or drugs, Heavy metals, CNS central nervous system

[12]. A number of validated assessment tools are available for screening, the grading of severity and to regularly monitor the illness. However, only a few have been validated for the palliative care setting, including the Confusion Assessment Method (CAM) [13] and the Memorial Delirium Assessment Scale (MDAS) [14]. The CAM is also the most widely used in palliative care and shows the best evidence due to its high sensitivity and specificity [15]. It consists of a nine-item questionnaire and four features diagnostic algorithm: acute onset and fluctuating course, inattention, disorganized thinking and altered level of consciousness. It requires prior training, takes around five minutes to complete and has to be combined with a test to assess cognitive impairment [16]. Although it is based on the
DSM-III-R, there might be a high agreement between the CAM and the DSM-5 [17].

\section{Consider predisposing and precipitating factors}

Delirium is an acute condition in which the identification and treatment of the underlying cause is crucial. While numerous predisposing risk factors such as age, male gender, sensory and cognitive impairments or brain tumours are known to increase the likelihood of disorder onset, precipitating factors like medication or metabolic factors as well as infections can trigger an episode [8]. Studies systematically assessing predisposing and precipitating risk factors in palliative care, especially the outpatient setting, are rare and their results vary. This can be explained by differences in 
the methodology as well as by the multifactorial and often nonspecific causes of the disorder. Palliative patients, who in most cases are severely multimorbid and terminally ill, are therefore particularly difficult to examine, since the identification of risk factors is sometimes masked, for example, by high systematic inflammation parameters [18].

As the aetiology is multifactorial and complex the use of guidelines, which will be displayed in more detail in the treatment section, as well as mnemonics like I WATCH DEATH (Infections, Withdrawal, Acute metabolic causes, Trauma, CNS pathology, Hypoxia, Deficiencies, Endocrinopathies, Acute vascular, Toxins or drugs, Heavy metals) are of help to identify the main risk factors in daily practice [19]. Furthermore, the consideration of the three "D"s: dementia, delirium and depression are essential, but also challenged due to an often-unknown cognitive status before admission as well as atypical or overlapping symptoms. However, generally speaking, in contrast to depression and dementia, delirium is characterised by a rapid onset, a high fluctuation of the symptoms during the day, a day night reversal and often a presence of vegetative symptoms.

\section{Focus prevention over treatment}

Since there are a number of guidelines, the ESMO Clinical Practice Guidelines on delirium in adult cancer patients can be specifically recommended for palliative settings as guidance in the differential diagnostic clarification [8]. Although management should primarily depend on the course of the disease, the patient's state and treatment goals, first and foremost, it should be the goal to prevent the occurrence of delirium. Therefore, it is advised to implement any measure that reduces mental or physical stress for patients such as supporting orientation (e.g. hearing/vision aids, tools for orientation in time and place, relative care treatment and family involvement), optimizing the sleep-wake cycle and keeping up mobility [20]. To train caregivers in preventive measures and in basic skills for the immediate detection of delirium is of particular importance in palliative home care settings, since only limited resources for care and diagnostics are available and any further changes in care setting such as the need for hospitalization represents additional risk in itself.

Furthermore, the consideration of the interplay between multimorbidity, frailty and polypharmacy seems to be of particular importance. Hence, paying attention to the medication and avoiding polypharmacy is of high relevance, as many drugs may cause or worsen delirium. Particular care has to be taken with benzodiazepines, opioids and corticosteroids and probably anticholinergics [8, 21]. A helpful tool for the evaluation of the delirogenic potential is the DEL-FINE score. It systematically categorizes substances and substance withdrawal in relation to their delirogenic potential in order to optimize the medication of vulnerable patients ranging from "three= strong delirogenic potential" to "zero = no delirogenic potential". A score of three is for example given to the medications: amitriptyline, atropine, clomipramine, orphenadrine, oxybutinin, scopolamine and the withdrawal of benzodiazepines, ethanol and opioids [22].

As opioids play a central role in palliative pain management, it is important to note that there is some evidence that switching the opioid to fentanyl or methadone may improve delirium [23]. Generally speaking, it is worth mentioning that opioids should not be used for sedation, which is a common mistake in clinical practice. Moreover, in case of an identified precipitating cause for delirium such as an infection or electrolyte imbalance, the initiation of adequate therapy according to the respective guidelines should be considered. The chosen treatment should be in concordance with the treatment goals of the patient and include an evaluation of the negative consequences of diagnostic procedures and the potential of reversibility.

Concerning delirium-targeted treatment, prevention by multicomponent nonpharmacological interventions seems to be a cost-effective way to significantly reduce delirium rates and falls. Nonpharmacological interventions address cognition, mobility, oxygen saturation, pain, hydration, sleep-wake cycle and sensory impairment and nutrition [20, 24]. Regarding pharmacological prevention, recent evidence casted doubt on the superiority of antipsychotics and other drugs to placebo [25-28]. Thus, there is only a recommendation for nonpharmacological interventions in people at risk according to the NICE guidelines [29]. Similarly, the evidence for antipsychotics in treatment of delirium is rather limited [25, 30, 31]. Aforementioned studies analysing prevention also did not find a significant improvement in treating delirium. However, the NICE guidelines still recommend short-term use of haloperidol if the patient poses a danger to others or themselves and nonpharmacological interventions have failed [20]. The efficacy is supported by a systematic review of haloperidol [32]. Contrarily, in a randomized clinical trial in a palliative setting haloperidol (and risperidone) worsened symptoms compared to placebo [28]. The ESMO guideline for delirium in cancer patients therefore advised against haloperidol and risperidone, although there is some evidence for olanzapine, quetiapine and aripiprazole to be potentially beneficial [8]. It should also be mentioned that the alpha-antagonist dexmedetomidine showed benefits in the ICU setting [33-35]. In conclusion, the value of antipsychotics and other pharmacological interventions is under debate and physicians should only use them with caution, as antipsychotics are among other things associated with prolongation of the QTc interval, extrapyramidal symptoms (EPS) and worsening of parkinsonian 
symptoms. However, when symptomatic treatment of agitation/hyperactivity, anxiety and distress is required, considerate usage of antipsychotics and even benzodiazepines, despite being delirogenic, may be useful.

In summary, rather than treat those already affected, physicians should focus on prevention, early detection and subsequent implementation of nonpharmacological interventions, since treatment options may not be as efficacious as formerly assumed. A practical guide to preventive, diagnostic and treatment measures is summarized in Table 2.

\section{Take home message}

- Beware of (hypoactive) delirium as it is rather common and easily missed.

- Target precipitating risk factors and avoid polypharmacy, in particular delirogenic drugs as benzodiazepines, opioids and corticosteroids.

- Use antipsychotics with caution and as short as possible.

Funding Open access funding provided by Medical University of Vienna.

Conflict of interest F. Portela Millinger and M. Fellinger declare that they have no competing interests.

Open Access This article is licensed under a Creative Commons Attribution 4.0 International License, which permits use, sharing, adaptation, distribution and reproduction in any medium or format, as long as you give appropriate credit to the original author(s) and the source, provide a link to the Creative Commons licence, and indicate if changes were made. The images or other third party material in this article are included in the article's Creative Commons licence, unless indicated otherwise in a credit line to the material. If material is not included in the article's Creative Commons licence and your intended use is not permitted by statutory regulation or exceeds the permitted use, you will need to obtain permission directly from the copyright holder. To view a copy of this licence, visit http://creativecommons.org/licenses/by/4.0/.

\section{References}

1. Watt CL, Momoli F, Ansari MT, Sikora L, Bush SH, Hosie A, et al. The incidence and prevalence of delirium across palliative care settings: a systematic review. Palliat Med. 2019;33:865-77. https://doi.org/10.1177/ 0269216319854944.

2. Salluh JIF, Wang H, Schneider EB, Nagaraja N, Yenokyan G, Damluji A, et al. Outcome of delirium in critically ill patients: systematic review and meta-analysis. BMJ. 2015;350:h2538-h2538. https://doi.org/10.1136/bmj. h2538.

3. de la Cruz M, Ransing V, Yennu S, Wu J, Liu D, Reddy A, et al. The frequency, characteristics, and outcomes among cancer patients with delirium admitted to an acute palliative care unit. Oncologist. 2015;20:1425-31.https:// doi.org/10. 1634/theoncologist.2015-0115.

4. Witlox J, Eurelings LSM, de Jonghe JFM, Kalisvaart KJ, Eikelenboom P, van Gool WA. Delirium in elderly patients and the risk of postdischargemortality, institutionalization, and dementia. JAMA. 2010;304:443. https://doi.org/10. 1001/jama.2010.1013.

5. Caplan GA, Teodorczuk A, Streatfeild J, Agar MR. The financial and social costs of delirium. Eur Geriatr Med. 2019; https://doi.org/10.1007/s41999-019-00257-2.

6. Finucane AM, Lugton J, Kennedy C, Spiller JA. The experiences of caregivers of patients with delirium, and their role in its management in palliative care settings: an integrative literature review. Psychooncology. : John Wiley and Sons Ltd;2017. pp. 291-300.

7. de la Cruz M, Fan J, Yennu S, Tanco K, Shin S, Wu J, et al. The frequency of missed delirium in patients referred to palliative care in a comprehensive cancer center. Support Care Cancer. 2015;23:2427-33. https://doi.org/10.1007/ s00520-015-2610-3.

8. Bush SH, Lawlor PG, Ryan K, Centeno C, Lucchesi M, KanjiS, et al. Delirium in adult cancer patients: ESMO Clinical Practice Guidelines. Ann Oncol. 2018;29:iv143-iv65.

9. Hosie A, Davidson PM, Agar M, Sanderson CR, Phillips J. Delirium prevalence, incidence, and implications for screening in specialist palliative care inpatient settings: a systematic review. Palliat Med. 2013;27:486-98. https:// doi.org/10.1177/0269216312457214.

10. World Health Organization. 6D70 Delirium. ICD-11 Mortal Morb. Stat. 2019. https://icd.who.int/browse11/1-m/ en\#/http://id.who.int/icd/entity/897917531. Accessed: 11.02 .2020

11. American Psychiatric Association. Diagnostic and statistical manual of mental disorders. 5th ed. Washington, DC: American Psychiatric Publishing; 2013.

12. Heymann A, Radtke F, Schiemann A, Lütz A, MacGuill M, Wernecke $\mathrm{K}$, et al. Delayed treatment of delirium increases mortality rate in intensive care unit patients. J Int Med Res. 2010;38:1584-95. https://doi.org/10.1177/ 147323001003800503.

13. Ryan K, Leonard M, Guerin S, Donnelly S, Conroy M, Meagher D. Validation of the confusion assessment method in the palliative care setting. Palliat Med. 2009;23:40-5. https://doi.org/10.1177/0269216308099210.

14. Lawlor PG, NekolaichukC, Gagnon B, Mancini IL, Pereira JL, Bruera ED. Clinical utility, factor analysis, and further validation of the memorial delirium assessment scale in patients with advanced cancer: assessing delirium in advanced cancer. Cancer. 2000;88:2859-67.

15. Shi Q, Warren L, Saposnik G, MacDermid JC. Confusion assessmentmethod: a systematic review and meta-analysis of diagnostic accuracy. Neuropsychiatr Dis Treat Dove Press. 2013;p:1359-70.

16. InouyeSK. Clarifying confusion: the Confusion Assessment method. Ann Intern Med. 1990;113:941. https://doi.org/ 10.7326/0003-4819-113-12-941.

17. Adamis D, Rooney S, Meagher D, Mulligan O, McCarthy G. A comparison of delirium diagnosis in elderly medical inpatients using the CAM, DRS-R98, DSM-IV and DSM-5 criteria. Int. Psychogeriatrics. 2015;27:883-9. https://doi. org/10.1017/S1041610214002853.

18. Maldonado JR, Kapinos G. Pathoetiological model of delirium: a comprehensive understanding of the neurobiology of delirium and an evidence-based approach to prevention and treatment. Crit CareClin. 2008;24:789-856.

19. Grassi L, Caraceni A, Mitchell AJ, Nanni MG, Berardi MA, Caruso R, et al. Management of delirium in palliative care: a review. Curr Psychiatry Rep. 2015;17:13. https://doi.org/ 10.1007/s11920-015-0550-8.

20. Delirium: prevention, diagnosis and management Clinical guideline Your responsibility. 2010. www.nice.org.uk/ guidance/cg. Accessed: 23.02.2020 
21. Caraceni A. Drug-associated delirium in cancer patients. Eur J Cancer Suppl. 2013;11:233-40. https://doi.org/10. 1016/j.ejcsup.2013.07.008.

22. Böhmdorfer B, Rohleder S, Wawruch M, van der Cammen TJM, Frühwald T, Jagsch C, et al. DEL-FINE: ein neues Tool zur Beurteilung der delirogenen Eigenschaften relevanter Arzneimittel für die Pharmakotherapie in Europa. ZGerontol Geriatr. 2016;49:416-22.

23. Mercadante S, Bruera E. Opioid switching: a systematic and critical review. Cancer Treat Rev. 2006;32:304-15. https:// doi.org/10.1016/j.ctrv.2006.03.001.

24. Hshieh TT, Yue J, Oh E, Puelle M, Dowal S, Travison T, et al. Effectiveness of multicomponent nonpharmacological delirium interventions: a meta-analysis. JAMA Intern Med. 2015;175:512-20.

25. Neufeld KJ, Yue J, Robinson TN, Inouye SK, Needham DM. Antipsychotic medication for prevention and treatment of delirium in hospitalized adults: a systematic review and meta-analysis. JAm Geriatr Soc. 2016;64:705-14.

26. Siddiqi N, Harrison JK, Clegg A, Teale EA, Young J, Taylor J, et al. Interventions for preventing delirium in hospitalised non-ICU patients. Cochrane Database Syst Rev. 2016;1. https://doi.org/10.1002/14651858.CD005563.pub3.

27. Oh ES, Needham DM, Nikooie R, Wilson LM, Zhang A, Robinson KA, et al. Antipsychotics for Preventing Delirium in Hospitalized Adults. Ann Intern Med. 2019;171:474. https://doi.org/10.7326/M19-1859.

28. Agar MR, Lawlor PG, Quinn S, Draper B, Caplan GA, Rowett D, et al. Efficacy of oral risperidone, haloperidol, or placebo for symptoms of delirium among patients in palliative care: a randomized clinical trial. JAMA Intern Med. 2017;177:34-42.

29. Young J, Murthy L, Westby M, AkunneA, O'Mahony R. Diagnosis, prevention, and management of delirium: summary of NICE guidance. BMJ. 2010;341:c3704-c3704. https:// doi.org/10.1136/bmj.c3704.

30. Nikooie R, Neufeld KJ, Oh ES, Wilson LM, Zhang A, Robinson KA, et al. Antipsychotics for treating delirium in hospitalized adults a systematic review. Ann Intern Med. 2019;171:485-94.
31. Wu Y-C, Tseng P-T, Tu Y-K, Hsu C-Y, Liang C-S, Yeh T-C, et al. Association of Delirium Response and Safety of Pharmacological Interventions for the Management and Prevention of Delirium. JAMA Psychiatr. 2019;76:526. https://doi.org/10.1001/jamapsychiatry.2018.4365.

32. Schrijver EJM, de Graaf $K$, de Vries OJ, Maier $A B$, Nanayakkara PWB. Efficacy and safety of haloperidol for in-hospital delirium prevention and treatment: a systematic review of current evidence. Eur J Intern Med Elsevier Bv. 2016;27:14-23. https://doi.org/10.1016/j.ejim. 2015.10.012.

33. Al-Qadheeb NS, Balk EM, Fraser GL, Skrobik Y, Riker RR, Kress JP, et al. Randomized ICU trials do not demonstrate an association between interventions that reduce delirium duration and short-term mortality. Crit Care Med. 2014;42:1442-54. https://doi.org/10.1097/CCM. 0000000000000224.

34. Reade MC. Low dose dexmedetomidine for the prophylaxis of perioperative ICU delirium - how much evidence is enough? J Thorac Dis. 2016;8:3020-3. https://doi.org/10. 21037/jtd.2016.11.30.

35. Reade MC, Eastwood GM, Bellomo R, Bailey M, Bersten A, Cheung B, et al. Effect of Dexmedetomidine Added to Standard Care on Ventilator-Free Time in Patients With Agitated Delirium. JAMA. 2016;315:1460. https://doi.org/ 10.1001/jama.2016.2707.

Publisher's Note Springer Nature remains neutral with regard to jurisdictional claims in published maps and institutional affiliations.

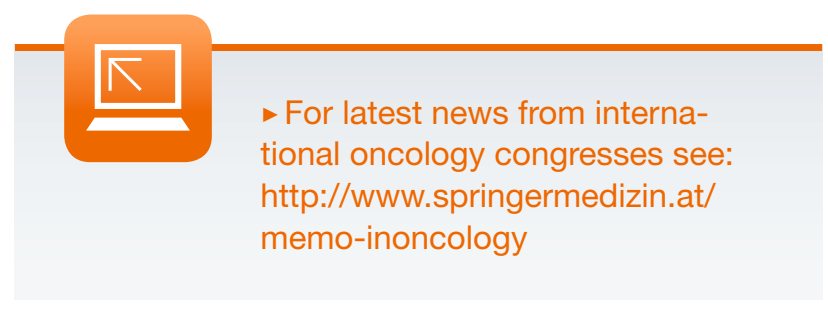

